\title{
Branding: A competitive strategy in the wine industry
}

\author{
B. Flores y M. Ortiz \\ Universidad de Guadalajara, Departamento de Negocios Internacionales y Mercadotecnia, Jalisco, México
}

\begin{abstract}
Resumen. A través de los años el vino ha tenido un desarrollo considerable en cuestión de competitividad dado que cada vez mas hay compañías que se unen a este mercado agroindustrial y es crucial el desarrollo de estrategias como el branding dirigido a la variante de país para que de esta manera se genere mayor apoyo y reconocimiento a nivel internacional de una buena tierra que cosecha vino de calidad. Por esto mismo, en el siguiente trabajo se podrá observar un marco teórico direccionado hacia la explicación de la competitividad de manera general junto con el desarrollo del concepto de branding con el especifico país, antes mencionada, para que de esta manera exista una contribución hacia la industria del vino, concepto que también es explicado de manera concisa para otorgar al lector un panorama de la importancia que tiene la relación del branding y la competitividad. Este trabajo se basa en un análisis cualitativo con bases en un estudio descriptivo- documental.
\end{abstract}

\begin{abstract}
Over the years the wine has had a considerable development in terms of competitiveness given that more and more companies are joining this agro-industrial market and it is crucial to develop strategies such as branding aimed at the country variant so that this In this way, greater international support and recognition of a good land that harvests quality wine is generated. For this reason, in the following work we can observe a theoretical framework directed towards the explanation of competitiveness in a general way together with the development of the concept of branding together with the country variant, mentioned above, so that in this way there is a contribution towards the wine industry, a concept that is also explained in a concise way to give the reader an overview of the importance of the relationship between branding and competitiveness. This work is a qualitative analysis based on a descriptive-documentary study.
\end{abstract}

Se dice que el vino no es un producto cuya fabricación en serie sea perfecta, ello se debe principalmente a que aun cuando las cepas de vides procedan de una cosecha adecuada, factores externos como el clima o el proceso de fermentación variarán de un año a otro, por lo que se podrá decir que la vid no es la misma [1], de tal forma que, a diferencia de otros bienes de consumo, donde el proceso puede tener variables más controladas, el proceso de elaboración de las bebidas fermentadas, especialmente del vino, no es rígido y aun cuando existen lotes amplios, su producción se encuentra más cercano al término artesanal que a una producción eminentemente industrializada.

Este hecho interviene directamente en la forma en que los vitivinicultores realizan la selección adecuada para la producción; la lógica de este hecho radica directamente en que el nivel de producción de las empresas es muy sensible a la capacidad instalada de una nación, no solo en términos de campos de vides, sino en el nivel tecnológico que éstas pueden tener [2], al mismo tiempo, el proceso puede verse influido directamente por la marca o el propio consumidor internacional, sin embargo, este hecho requiere que se genere una construcción internacional de marca, lo cual, es conocido como branding, de tal manera que los consumidores, sin importar en qué parte del mundo se encuentren, sean sensibles a los procesos y no a las variaciones del vino existentes por su tipo de producción [3].

Es así como una organización dedicada a la producción de vinos, debe tener la capacidad de manejar un entorno cambiante, de tal forma que pueda adaptarse continuamente a las modificaciones del ambiente internacional y lograr establecer estrategias que promuevan ventajas reales y sostenibles, de tal manera que se induzca a la competitividad, sin embargo, el hecho se encuentra en que éstas deben visualizarse desde el entorno angular del cambio de los negocios internacionales, dado que, los hechos comerciales moldean directamente hacia donde se debe enfocar el recurso.

La investigación presentada analizará el desarrollo de los mercados internacionales y su posición determinante en la configuración de estrategias para generar una identidad de marca, es decir branding internacional, a fin de aprovechar los canales y habilidades efectivas de posicionamiento, variables manejadas por Orth, Lockshin y Hauteville [4]; de tal manera que provean de un enfoque competitivo desde la perspectiva de la innovación y los factores productivos [5].

\section{La industria vitivinícola}

De acuerdo con Bărbulescu [6] la tradición vitivinícola se remonta desde los antiguos tiempos de Europa alrededor de 6000 años y así mismo Europa mediterránea donde el vino fue parte fundamental en el consumo [7-9]. Y actualmente el vino se ha extendido en los últimos años debido a su diversidad donde enólogos y productores han centrado sus esfuerzos en el desarrollo de esta bebida [10] para que esté disponible en todo rango de clases sociales y su producción gracias a la globalización ha ido en aumento, desde un proceso artesanal hasta un proceso 
industrial magnificado a grande escala [11] y este producto agroindustrial es altamente sectorial en cuestión de su país de origen y su proceso industrial de preparación para su producto final [12].

Así mismo, autores como Orth, Lockshin \& D‘Hauteville [4] determinan esta división como; viejo mundo y el nuevo mundo. Por un lado, la primera división está enfocada a las naciones que han desarrollado el vino como una manera competitiva desde tiempos de antaño, cuyo valor ha sido alto a través de los años y su posicionamiento tanto de marca como de país de origen ha sido de manera estratégica, estos países son Francia, España e Italia [13].

Por otra parte, el nuevo mundo tiene las características de naciones que han trabajado por desarrollar ventajas competitivas en la calidad y la innovación con nuevas cepas y nuevas maneras de generación de vino para que con esto puedan tener lugar dentro de los rankings de la industria del vino, estos países son Australia, Argentina, Chile, Nueva Zelanda, Sudáfrica y los Estados Unidos en específico el estado de California [14]. Debido a esta división, la producción de vino se ha incrementado rápidamente y la innovación ha ocupado un lugar especial dentro de cada empresa e incluso del país para ser competitivo y alcanzar un negocio exitoso [2].

De hecho, la entrada de nuevos competidores a la industria del vino en todo el mundo plantea preguntas importantes sobre qué estrategias tomar para ser competitivas [15]. Además, la innovación en los procesos del vino como ya se mencionó ha desarrollado el aumento de la producción en las empresas establecidas y una fuerte fuerza de comercialización de los nuevos participantes; países que anteriormente no producían vino y ahora lo hacen. Por lo tanto, las empresas vinícolas deben de buscar una continuación directa y lógica de la competitividad adecuada para la adaptación del mercado cambiante y este incluye una calidad alta en la producción, el cumplimiento de los precios y las herramientas para llevar esta producción al consumidor final [2].

\section{La construcción de marca - branding}

Una marca de acuerdo con Aaker [16] evoca a la esencia de un producto o servicio que penetra la mente del consumo, así mismo Rowley [17] opina que una marca representa la simplificación para un consumidor cuando decide que comprar ya que la misma ya le ha proporcionado información anteriormente. Por lo tanto, es un punto importante para la marca la distinción y la información de los atributos de su producto o servicio al segmento de mercado al que está dedicado [18].

El branding también está asociado con la creación de valor hacia la oferta persuasiva para el cliente y su satisfacción [19]. Así mismo Cortázar [20] manifiesta que posicionamiento está relacionado a la percepción de los clientes y su pensamiento, aunado con la opinión de Davis (2000) donde considera este concepto como un lugar dentro de la mente del consumidor que se gana por medio de la capacidad de ser diferente a los competidores donde Kapferer [21] considera que es el principio de la competencia entre empresas y así ser valorado por los consumidores.

Y todo esto sucede gracias a que hoy en día, la elección por marcas por parte de los consumidores ha construido el benéfico esfuerzo de las empresas por realizar estrategias para su posicionamiento [22,23]. Sin embargo, el consumidor actual también está enfocado en recibir una experiencia por parte de la marca, es decir esa necesidad de tener una sensación palpable por parte de la marca y con esto contribuir a su comportamiento hacia ella; una fidelidad [24]. Esta experiencia se ve reflejada en la fuerza de ventas de la empresa, así como los patrocinios, puntos de ventas entre otras cosas, por obvias razones esto provoca en la compañía un fuerte análisis de crear la experiencia única hacia los consumidores para retener su confianza y por supuesto su fidelidad mediante la diferenciación de su marca para competir de manera efectiva a la competencia [24-28].

A manera de explicar a mayor rasgo la diferenciación de marca, pudiera ser útil hablar acerca de la paridad de marca y la similitud de marca, en donde cabe destacar que este derivado no funciona en todos los productos de acuerdo a Bialdyga \& Britzelmaier [29]. Por lo tanto Iyer $\&$ Muncy [30] describen la paridad de marca como la percepción del consumidor de que un producto y otro son tan cercanos que una diferencia latente entre uno y otro es muy reducida. Y Bijmolt, Wedel, Pieters, \& Desarbo [31] explican la similitud de marca como el panorama en donde los productos pueden ser cercanamente similares pero así mismo tienen valores y cualidades que provoca una diferenciación latente entre un producto y otro.

En el posicionamiento de marca existen niveles para identificar, separar y analizar cómo se desarrolla de distintas formas la marca para ser competitiva en el mercado; Marca Base, Posicionamiento de Sector, Posicionamiento de País y Posicionamiento Global [32]. Cabe aclarar que el tercer nivel será el que se desarrollará en este trabajo presentado. Entonces, el primer nivel; la marca base es en función de la exportación de los productos y la manera efectiva de diferenciar el producto utilizando en su mayoría el marketing mix (Precio, Promoción, Plaza, Producto), pero solo en función de la marca [33]. Por explicación del segundo nivel; Posicionamiento de Sector, se refiere al valor agregado (ventaja competitiva) que tiene una asociación de empresas de un solo sector para ganar más competitividad a nivel internacional [34].

Pasando el tercer nivel ya que este será explicado de mayor magnitud, el cuarto nivel; marca global, se refiere a un traspaso de que la marca sea de un país solamente, se caracterice por su país de origen sino que el mismo valor de marca ha sido tan alto que la misma marca es el centro sin necesidad de revisar el país o desarrollo del país [31].

\section{Posicionamiento de país o place branding}

Este término ha sido utilizado desde varios años atrás pero con un objetivo diferente a través de los años [32], este hace referencia de un territorio de un país o el mismo país que contiene tal fuerza de marketing y de consumo en donde regiones, ciudades o países han utilizado [33]. De acuerdo con Herget, Petrú y Abrhám [35] hay suficiente motivación para una ciudad, una región o un país de generar su propia marca país, por los múltiples beneficios que esto conllevaría; como inversión y aumento de turistas.

El desarrollo de esta variante de branding ha llevado al Estado a generar estrategias diversas para competir con 
otras regiones y países con el objetivo de que cada vez más el territorio propio sea más atractivo [36,37] Aunando mas en como lo describe Kotler [37] estas actividades tienen el objetivo primordial de generar una mejor calidad de vida entre la población de la región, por lo tanto Kavaratzis, Warnaby y Ashworth [38] definen esta estrategia de marca país como la percepción generada en los posibles consumidores con la intención de la permanencia en la mente del mismo y su completa satisfacción. Claro está, que la construcción de una imagen positiva genera desarrollo económico y como ya se menciono, calidad de vida satisfactoria, en contrario, un error en la construcción de la marca país puede provocar daños perjudiciales hacia el país y lograr cambiar ese error costaría años revertirlo [36].

Por lo tanto, esta estrategia para algunos sectores de ciertas industrias genera grandes ventajas competitivas ante los demás competidores y para la industria del vino en particular, gracias a la generación de esta estrategia de branding que se ha construido en ciertos países poco a poco ha sido pieza clave para una óptima penetración de la mente del consumidor.

\section{Competitividad internacional}

El concepto de competitividad en estos días ha sido una de las palabras más utilizadas en el campo económico y se ha convertido en un concepto ambiguo debido al múltiple análisis que se ha tenido durante estos años $[39,40]$. Según Nedić, Lepojević, Despotović \& Cvetanović, [41] la competitividad se refiere a la capacidad de individuos, empresas, comunidades, países y regiones para competir y ser mejores, más innovadores y más creativos que otros interesados en el ámbito local, nacional, regional y global; en la creación de valor agregado, y esto comenzó porque según Kharub [48] "las estrategias industriales tradicionales son cada vez menos efectivas" y la competitividad ha contribuido a cambiar el entorno empresarial para mejorar en todo el mundo.

Por esta razón, ha habido numerosos estudios sobre la competitividad que existe en las naciones para explicar por qué algunas naciones crecen significativamente y otras simplemente se mantienen en el camino en relación con su tasa de crecimiento y desarrollo como país [42]. Ya que donde hay un crecimiento económico y productivo en un país se ha demostrado la importancia de la competitividad misma [43].

El concepto de competitividad se ha desarrollado durante más de dos décadas; donde se ha convertido en uno de los principales objetivos para las empresas y la búsqueda constante por parte de los territorios de los países [44] donde la localización como países tiene demasiado campo para analizar porque según Bilgen [45] los países usan la competitividad para figurar en el ranking de posición global según su ventaja competitiva en cada industria que desarrollan gracias a estrategias donde junto con el concepto de globalización y la apertura de los mercados ha facilitado el comercio internacional [46], reduciendo las barreras entre los países.

Para definir la competitividad a grandes rasgos, Delgado, Porter y Stern [42] mencionan que la competitividad tiene la expectativa de obtener un nivel de producción sostenida para que con esto, una persona capaz de trabajar sea aceptada por su calidad y con esto contribuya a
Tabla 1. Elaboración de la autora con datos teóricos [47].

\begin{tabular}{|l|c|l|c|}
\hline $\begin{array}{l}\text { País de } \\
\text { Origen }\end{array}$ & Posición & $\begin{array}{l}\text { Sector al que } \\
\text { pertenece }\end{array}$ & $\begin{array}{l}\text { Porcentaje } \\
\text { de Mercado }\end{array}$ \\
\hline Italia & $1^{\circ}$ & Viejo Mundo & $41.8 \%$ \\
\hline Francia & $2^{\circ}$ & Viejo Mundo & $16.41 \%$ \\
\hline España & $3^{\circ}$ & Viejo Mundo & $17.72 \%$ \\
\hline Sudáfrica & $4^{\circ}$ & Nuevo Mundo & $5.68 \%$ \\
\hline Estados Unidos & $5^{\circ}$ & Nuevo Mundo & $3.66 \%$ \\
\hline Chile & $6^{\circ}$ & Nuevo Mundo & $3.2 \%$ \\
\hline Argentina & $11^{\circ}$ & Nuevo Mundo & $0.3 \%$ \\
\hline Nueva Zelanda & $12^{\circ}$ & Nuevo Mundo & $0.2 \%$ \\
\hline
\end{tabular}

construir un negocio rentable. y negocios competitivos. Esto genera un mayor nivel de ingresos en las empresas que si se mantienen en el orden correcto y se priorizan desde el individuo hasta el general, las empresas, las industrias, las regiones y los países pueden ser sostenibles.

\section{Metodología y resultados}

En la parte metodológica hasta este punto del estudio es documental y es parte de una investigación más grande de tesis de maestría. Es documental dado que como prioridad es evaluar al mercado de manera teórica sustentada por diversos autores que hablan y discuten acerca de la importancia de este tema para contribuir a la industria del vino. Ya que muchos países han trabajo de manera sistemática en las últimas décadas para posicionar su marca país y sus vinos por ende. Como se observó en la parte teórica, la generación de la marca como país es uno de los pilares importantes para generar estrategias competitivas efectivas en un mercado tan competido hoy en día.

Es importante destacar que el objetivo que se tiene con esta investigación de maestría es aportar estrategias para la industria misma de México. Si bien como se puede observar en la siguiente tabla, México aún ni siquiera figura de manera teórica en las evaluaciones de mercado.

Debido a los resultados que se encuentran en la teoría y se presentan en la Tabla 1, los países que se mencionaron en la teoría como principales del sector tanto viejo o nuevo mundo, están dentro del ranking principal de mercado compartido según los autores [47].

Así mismo este estudio documental es de carácter cualitativo dado que como lo menciona Herrera (2008) es la variante de la investigación que extrae descripciones de un fenómeno o problema mediante acciones diversas cuyos métodos son fundamentales, para una especificación de este trabajo se puede observar como método de una investigación cualitativa una teoría fundamentada con la variable branding y su explicación conceptual para una generación posterior de estrategias para la industria.

Como aportación hasta el momento por parte de la recolección de datos propios de la investigación cualitativa, las variables desarrolladas a lo largo de este documento; Competitividad y Branding que influyen a la industria vitivinícola.

También se puede apreciar como punto medular dentro de lo analizado por medio de la teoría, el desarrollo de una marca fuerte hacia el mercado para que con esto 
exista una fidelidad y creación de lealtad del consumidor y por esto mismo se aborda el branding como punto clave a la competitividad de esta industria ya que es punto fundamental el establecimiento de la marca hacia la mente y preferencia del consumidor avalada en este caso por el país de origen, ya que dentro de esta industria, esta mecánica en donde el país de origen contribuye en gran medida hacia el posicionamiento de marca es un detalle importante que tomar en cuenta cuando se analiza alguna estrategia, por la necesidad de una colaboración del estado o región involucrada. Por lo tanto es fundamental una presentación de un estudio teórico y documental en primera instancia para entender el funcionamiento y modus de esta industria.

A manera de conclusión se puede observar que la industria del vino ha sido dinámica y evolutiva a través de los años lo cual ha dado oportunidad de un análisis profundo de cómo ha funcionado y como se han posicionado año con años las marcas más fuertes alrededor del mundo. Como lo menciona Diaz Osorio, Valdes, \& Hernandez [47], en su análisis dirigido al mercado chileno en cuestión del vino, expresa la funcionalidad de una generación de marca posicionada a nivel internacional donde equivale al país el $6.15 \%$ de la exportación del país en total, el branding a nivel país se ve claramente como una estrategia eficiente para esta industria vitivinícola. Así mismo en su estudio de Lacerta Neacsu \& Madar [1] comparando esta marca para otorgar estrategias al mercado del vino rumano donde llega a una conclusión intermedia entre las áreas de oportunidad y las amenazas que se presentan para el desarrollo de Rumania en este sector.

Así mismo por otro lado Doloreux \& Lord-Tarte, [5] definen el desarrollo de la industria del vino en una parte fundamental, la innovación en el proceso del vino para un mejor posicionamiento de las marcas artesanales de la región de Columbia Britania en Canadá y aunado a esto Overton \& Murray [10] comentan otra variable importante que han observado en la industria vitivinícola en Nueva Zelanda y es el capital tanto de conocimiento como financiero en cuestión de contribuir a la industria y como se pudo observar en el marco teórico, Nueva Zelanda forma parte del segmento del Nuevo Mundo y esta posicionado como una marca país fuerte en cuestión de valor y volumen.

Por lo tanto hasta donde el estudio documental ha otorgado información la industria del vino en México tiene grandes oportunidades en cuestión de desarrollo de branding nivel país para que con esto se pueda llevar sinergia en el mercado internacional de esta bebida.

Es importante destacar que hasta este momento es un estudio preliminar documental antes explicado en la sección de metodología cuyo objetivo es presentar la fundamentación teórica y describir la situación actual de la industria del vino, el siguiente paso, solo con objetivo de presentársela al lector, es generar un instrumento de medición hacia el branding y la relación con la competitividad para la evaluación de la industria en México, ya teniendo fundamentación en otras partes del mundo donde el vino ha sido pieza clave de desarrollo, es una posibilidad que en México también se pueda establecer un modelo en donde se pueda posicionar como país con alta calidad en su vino.

\section{Referencias}

[1] N. Neacsu, A. Madar, Case Study?: Lacerta Winery 9 (2016)

[2] S. Bondarenko, V. Nizyayeva, Econ. Processes Manage. 2 (2017)

[3] A. Heijbroek, Wine is Business. Shifting Demand and Distribution: Major Drivers Shaping the Wine Industry. Utrecht: Rabobank International (2003)

[4] U. Orth, L. Lockshin, F. d'Hauteville, Int. J. Wine Business Res. 39, 5 (2007)

[5] D. Doloreux, E. Lord-Tarte, Euro. Planning Stud. 22, $1062(2014)$

[6] O. Bărbulescu, Potential and Risks in the Rom. Wine Ind. 10 (2017)

[7] T. Unwin, El vino y la viña. Geografía Histórica de la Viticultura y el Comercio del Vino (Tusquets, 2001)

[8] J. Sánchez, El Valor Social y Territorial del Vino en España (La economía del vino en España y en el mundo, 2014), p. 31

[9] E. Fernández, V. Pinilla, Historia económica del vino en España (1850-2000), (La economía del vino en España y en el mundo, 2014), p. 67-98

[10] J. Overton, W. Murray, G. Banks, The Race to the Bottom of the Glass? Wine, Geography, and Globalization (Routledge, 2012), vol. 9, p. 273

[11] G. Campbell, N. Guibert, Wine, Society and Globalization: Multidisciplinary Perspectives on the Wine Industry (New York and Basingstoke, Palgrave MacMillan, 2007)

[12] J. Overton, W. Murray, Geographical J. 182, 49 (2016)

[13] I. Bardají, Distribución y Consumo 78 (2004)

[14] P. Jenster, D. Smith, D. Mitry, I. Jenster, The Business of Wine. A Global Perspective (Copenhagen Business School Press, 2008)

[15] L. Saglietto, F. Fulconis, D. Bédé, A. Goes, R. Forradellas, Wine industry supply chain: international comparative study using social networks analysis 17, 55 (2016)

[16] D. Aaker, Managing Brand Equity: Capitalising on the Value of a Brand Name (Free Press, 1991)

[17] J. Rowley, Inf. Rev. 28, 131 (2004)

[18] J. Rekom Van, P. Verlegh, R. Slokkers, J. Business Res. 62, 339 (2009)

[19] S. Goswami, IUP J. Brand Manag. 12, 45 (2015)

[20] L. Cortázar, Revista Lasallista de Investigación 14 (2017)

[21] J. Kapferer, Strategic Brand Manage (The Free Press, 1992)

[22] A. Ismail, T. Melewar, L. Lim, A. Woodside, Mark. Rev. 11, 205 (2011)

[23] L. Zarantonello, B. Schmitt, J. Brand Manage. 17, 532 (2010)

[24] J. Brakus, B. Schmitt, L. Zarantonello, J. Mark. 73 (2009)

[25] L. Labrecque, G. Milne, Mark. Lett. 24, 165 (2013)

[26] R. Makadok, D. Ross, Strategic Manage. J. 34, 509 (2013)

[27] G. Carpenter, R. Glazer, K. Nakamoto, J. Mark. Res. 31 (1994)

[28] A. Ehrenberg, N. Barnard, J. Scriven, J. Advertising Res. 37, 7 (1997) 
[29] D. Bialdyga, B. Britzelmaier, Int. J. Manage. Cases 13 (2011)

[30] R. Iyer, J. Muncy, J. Advertising Res. 45, 222 (2005)

[31] T.H. Bijmolt, M. Wedel, R. Pieters, W. Desarbo, Int. J. Rese. Mark. 15 (1998)

[32] L. Lazo, Contabilidad Y Negocios 1, 36 (2012)

[33] M. Carroll, S. Nelson, Aust. J. Reg. Stud. 23 (2017)

[34] A. Wæraas, H. Bjorna, T. Moldenaes, Public Manage. Rev. 17, 1282 (2015)

[35] J. Herget, Z. Petrù, J. Abrahám, Economics and Sociology 8, 119 (2015)

[36] V. De Elizagarate, Marketing de Ciudades: Estrategias Para el Desarrollo de Ciudades Atractivas y Competitivas en un Mundo Global (Ediciones Pirámide, 2008)

[37] P. Kotler, Presentation on Marketing Places, Portimao International Conference on Marketing Places (Portimao, 24 de junio 2009)

[38] M. Kavaratzis, G. Warnaby, G. Ashworth, Rethinking Place Branding: Comprehensive Brand Development for Cities and Regions (Heidelberg, Springer, 2015)
[39] A. Ajitabh, K. Momaya, Competitiveness of Firms: Review of Theory (Frameworks and Models, 2003)

[40] A. Jambor, A. Toth, D. Koroshegyi, AGRIS on-line Pap. Economics and Informatics 9, 27 (2017)

[41] V. Nedić, V. Lepojević, D. Despotović, D. Cvetanović, Clanci. 2, 278 (2015)

[42] M. Delgado, M. Porter, S. Stern, Clusters, Convergence and Economic Performance (US Census Bureau Center for Economic Studies, Cambridge, 2012), p. 1

[43] C. Ketels, J. Industry Competition and Trade 6, 115 (2006)

[44] L. Constantinescu, Int. Comp. Manage. 18, 326 (2017)

[45] H. Bilgen, A. Varoglu, Competitiveness Rev. 26, 537 (2016)

[46] F. Blanco-Mesa, A. Gil-La Fuente, Econ. Comput. Econ. Cybernetics Stud. Res. 51, 231 (2017)

[47] J. Díaz, R. Valdés, N. Hernández, A positioning mapping approach from Germany 47 (2015)

[48] M. Kharub, R. Sharma, Competitiveness Rev. 27 (2017) 